The application of the results of the fundamental work on nucleation to practical problems was well illustrated, particularly in connexion with the soundness of iron castings. Increasing the degree of eutectic nucleation increases the tendeney to shrinkage defects, and methods for reducing eutectic nucleation are being tried. The laboratory findings have been well confirmed by industrial trials.

Fundamental work is also proceeding on the interplay of thermal and nucleation effects in the production of chilled castings, and also on the mechanism of solidification of white cast irons in which the eutectic of austenite and iron carbide can appear in various patterns related to the nucleation of the melt and the amount of undercooling before solidification. The detection of eutectic cells in white cast irons has proved difficult and the use of the reflecting polarizing maicroscope has so far proved to be the most useful tool.

The mechanism of the corrosion attack on cast iron in diesel engine waterways has been studied and the special test rig used was demonstrated. Evidence at present shows this to be caused by the accumulation of acidic oxidation products of the glycol in the coolant, the chief of which is formic acid. The formation of formic acid is probably accelerated by the loss of the copper corrosion inhibitor.

The problem of phosphorus in foundry pig iron was illustrated. To a large extent British iron ores of low phosphorus content are exhausted, and foundry pig irons produced from home ores generally contain more than 1 per cent phosphorus. This element has many harmful effects in cast iron when present in such amounts and the Association has just completed an extensive survey of the possibility of utilizing high phosphorus iron ores for the production of low phosphorus foundry pig iron. It has been possible to demonstrate that by top-blowing with oxygen in a rotary Kaldo converter, the phosphorus of phosphoric pig iron can be substantially eliminated and the iron cheaply recarburized.
Cast iron in steam engineering applications is generally limited to temperatures not exceeding $450^{\circ} \mathrm{F}$. The Association has just completed the first part of an investigation showing that this is an unrealistic limitation since almost all cast irons have dimensional stability up to at least $750^{\circ} \mathrm{F}$. Creep tests are in progress to provide additional evidence.

Cast iron is not a truly elastic material. When stressed the strain can be shown to involve a recoverable anelastic component and an irrecoverable component. By a study of the stress/strain curve the latter has been shown to involve true plastic deformation and also a mechanism by which the graphite voids are increased in size. The application of triaxial stress systems by means of mild notches is shown to modify the mechanical properties. Typical results obtained in this investigation were demon. strated.

For many years the Association has been studying the influence of the gaseous elements in cast iron, and the practical implications of this work were illus. trated. In particular, the influence of aluminium in cast iron in causing the decomposition of water vapour, leading to the solution of hydrogen, was emphasized with many industrial examples.

The Association maintains a Foundry Operations Section to provide the iron-founding industry with an advisory service on productivity and working efficiency. Recently, considerable interest has been displayed in a form of time-lapse ciné photography known as 'Memo-motion' and the equipment used and typical results obtained on foundry operations were demonstrated.

The exhibition material was designed to demonstrate that the work of the Association involved largely applied research undertaken specifically in support of the iron-founding industry, its materials, processes, working conditions and productivity. Many examples were given illustrating how the worker in a more or less fundamental field could receive inspiration and ideas by contact with the day-to-day problems of industry.

H. Morrogh

\title{
ATOMIC MECHANISMS OF FRACTURE
}

A CONFERENCE on "The Atomic Mechanisms of Fracture" was held at Swampscott, near Boston, Massachusetts, during April 12-14, organized by the National Academy of Sciences--National Research Council. More than 400 people attended, including about twenty from overseas, and twentyfive papers were presented.

Although the main emphasis was on the properties of metals, there were a number of papers dealing with non-metallic crystals, and non-crystalline solids. In the last category interest centred on dynamic effects : H. Schardin presented some rather precise results on the measurement of crack velocities in glasses of various compositions which showed that, although it is approximately true that the maximum crack velocity is proportional to the speed of longitudinal elastic waves, there are significant discrepancies which appear to be correlated with the chemical constitution of the material. H. Kolsky discussed the similarities in behaviour of plastics and viscous liquids when subjected to tensile-stress pulses of short duration, caused by the stress waves from an explosion. At the other extreme end of the time- scale, R. J. Charles discussed the dependence upon time of the strength of silicate glasses under static loading. Attributing this to the chemical action of atmospheric water vapour at the tip of a crack, he adduced supporting evidence from the behaviour of crystalline oxides under similar conditions.

In the main field of interest of the conference, it was clear that the complexity of the process of fracture is now agreed. Four types can usefully be distinguished : (1) ductile; (2) brittle; (3) creep; (4) fatigue. It should, however, be emphasized that this is no more than a classification of convenience; each heading probably covers a variety of processes ; and when any particular body changes from one piece into two pieces a selection of these processes may have been involved, according to the conditions of the experiment. An extreme case arises when a crystal of a soft metal draws down, in tension, to a chisel edge or a point. The mechanism, doubtfully included under the general heading of fracture, is the flowing of material away from the developing neck, by single or multiple glide processes. It was suggested that the central, fibrous part of the typical 
cup-and-cone tensile fracture of, say, a copper bar might be essentially similar to this. A paper by C. Crussard et al. emphasized the value of the electron microscope in the study of such fracture surfacesa value which arises not so much from its high resolving power as from its great depth of focus. Crussard showed that in such a fibrous ductile fracture there is evidence of repeated nucleation of new cracks ahead of the growing tip of the major crack, such nucleation taking place usually at minute inclusions in the metal. The new cracks may then join with the major crack by a process of repeated necking down, as just sug. gested.

R. W. K. Honeycombe and C. J. Beevers showed that, by suitable choice of conditions, such necking can be suppressed, even in single erystals of a facecentred cubic material, and that when this is done one obtains a mode of separation which can be more properly called a true ductile fracture, the separation of the two parts taking place along a previously heavily deformed glide plane and being apparently controlled by the resolved shear stress on this plane. On the other hand, single crystals of iron, tested at low temperatures by N. P. Allen and B. Edmondson, either neck down to 100 per cent reduction of area, as already described, or else cleave along the $\{100\}$ cleavage plane without obvious prior slip. The choice between the two modes of behaviour is determined by the direction of the tensile stress relative to the crystal axes, and the transition is quite sharp.

The topic which received the greatest amount of attention during the conference was the well-known ductile-brittle transition in polycrystalline iron which normally takes place rather below room temperature. A paper by G. T. Hahn, B. L. Averbach, M. Cohen and W. S. Owen reported an extensive series of observations on the tensile fracture of mild steels, of various compositions and grain sizes, over the temperature range $20-290^{\circ} \mathrm{K}$., which showed that the phenomena are more complex than had perhaps previously been realized. In particular, the authors claim that different processes are important in different ranges of temperature--other things being equal-and that mechanical twinning plays a decisive part at the lowest temperatures.

Microscopic observations of specimens broken, or almost broken, under conditions near those obtaining at the brittle-ductile transition showed the existence of numerous micro-cracks, each usually confined to a single grain. The frequency of occurrence of such cracks varied systematically with the conditions of the test and the authors maintain that it is necessary to subdivide the process of fracture in this transition range into: (a) true initiation in which some plastic deformation is probably essential, (b) growth within the original grain, $(c)$ propagation through the rest of the specimen. A useful concept which arises is that the 'effective' value of the surface energy of the newly formed surfaces may be much larger - the authors deduce 10 times largerfor $(c)$ than for $(b)$. This is indeed reasonable in the light of some of the fractographic studies of J. R. Low, which show very clearly the change of character of a cleavage surface which can take place when the crack passes from one grain into another less favour. ably oriented for cleavage.

A contribution from N. J. Petch summarized his own extensive work, relevant to the more metallurgical aspects of the same problem. In addition to the grain size, temperature, and strain-rate, which are commonly recognized as important variables, he discussed also the mechanisms by which the carbon, nitrogen and other elements commonly present in steels affect the various stages of the fracture process, and the ways in which their influence can be modified by previous mechanical and thermal treatment. The introductory paper by $\mathrm{A}$. H. Cottrell also dealt with the brittle-ductile transition at some length, in addition to giving a general survey of the whole subject of the conference. Although some points of controversy remain, and although much detailed work remains to be done, it appears that the broad outlines of the explanation of the ductile-brittle transition are becoming settled.

The two main contributions on creep fracture, namely, on slow fracture at temperatures which are high relative to the melting point, came from $N$. J. Grant and R. C. Gifkins. The features which distinguish this type of failure from the others are the considerablo importance of grain-boundary sliding and grain-boundary migration, the comparative ease of dislocation climb processes, and the possibility of deformation by the migration of point defects under the action of stress. It was made very clear that these four processes can be inter-related in several ways, and that they can all be inter-related to any deformation by dislocation glide which may be taking place in the body of the grains. Failure is often, but not always, intergranular, and is often, but not always, associated with the presence of 'voids' in the grain boundaries. The fracture behaviour was reported to be particularly sensitive to the presence of small amounts of impurity in a nominally pure metal. The paper by Gifkins summarized some of the earliex experimental work, which is considerable in quantity and not always self-consistent. It is clear that it is likely to be some time before the present confusion approaches anything approximating to a unified body of knowledge, although the general lines of the pattern are beginning to emerge.

The problem of fracture during fatigue is perhaps in the least satisfactory state of all. Of the papers presented at the conference, most were concerned with the early stages of the process. W. A. Wood described the interesting results obtained by a taper sectioning method applied to a partially fatigued copper specimen. As with so many other papers on other topics, the impression given by this work is that the mechanism of fracture is more complex than had hitherto been supposed. The emphasis is on the events taking place close to the surface of the solid, and leading up to the formation of a true crack. E. S. Machlin and A. J. McEvily deseribed experiments on four inorganic crystals which seem to show an inter-relation between liability to fatigue fracture and the ability of the crystal to become deformed by cross-slip; the possibility of the forma. tion of 'extrusions' from the metal surface also seems to be correlated with both these features. A similar point emerges from a comparison of two papers on copper by W. A. Backofen and N. Thompson, respectively, one of which dealt with the effect of erystal orientation on liability to fracture while the other related crystal orientation to extrusions.

Mention must also be made of the contribution by E. R. Parker on the cleavage fracture in tension of single crystals of magnesium oxide. These observations, and similar work by Stokes et al., mentioned in the course of discussion, demonstrate the advantages to be gained by experimenting on ionie rather than metallic crystals, and emphasize a point that was made on a number of occasions. This is that, on 
close examination, no crystal breaks in a truly brittle manner; the fracture is always preceded by some small amount of plastic deformation. This is one of the key points in connecting theories of fracture with current views on the mechanical behaviour of crystalline solids. The other general feature of the proceedings, already mentioned, was the evident fact that in no circumstances is fracture a simple process. The realization that "when a problem is difficult, it is probably two problems" is perhaps the most important step on the road to a solution.

N. THOMPSON

\section{OXIDATION OF ORGANIC COMPOUNDS}

A SYMPOSIUM on the oxidation of organic compounds was held in the Stern Hall at Queen Mary College, University of London, during April 13-14.

In his opening address Mr. D. A. C. Dewdney, director of Esso Petroleum Co., Ltd., spoke of the importance of a free exchange of scientific information and the need of still further fundamental research into basic problems; an improving standard of living and higher productivity are largely dependent on the commercial application of original scientific discoveries. In welcoming visitors to the symposium, he referred especially to those from the U.S.S.R. and Czechoslovakia.

The first part of the scientific discussion was concerned with the course of the oxidation of saturated hydrocarbons by chromic acid in acetic acid contain. ing some sulphuric acid. From the papers and the discussion which developed there was general agreement that the first recognizable stage in the oxidation is hydrogen abstraction from the hydrocarbon, and that the factors which influence the speed of the reaction are mainly steric and configurational. The same factors also determine the rate of oxidation by chromium trioxide in anhydrous media.

Dr. J, Roček (Institute of Chemistry, Czechoslovak Academy of Science, Prague) reported that $n$-paraffins are oxidized at a rate directly proportional to the number of methylene groups, the rate constant for any individual member being $k_{n}=k_{\mathrm{CH}_{2}}(n-2)$, where $n$ is the number of carbon atoms and $k_{\mathrm{CH}_{2}}$ the rate constant for the oxidation of a single methylene group. The relative rates of oxidation of the methyl, methylene, and methine groups in open chain hydrocarbons have been found to be $0.015: 1: 32-77$. Measurements of the rate of oxidation of cycloalkanes disclosed some interesting anomalies.

The oxidation-rate of the tertiary $\mathrm{CH}$-group is found to vary somewhat with the bulk and structure of the neighbouring alkyl groups; these variations are due mainly to polar factors and are similar to changes in rates of solvolysis of the corresponding tertiary chlorides. Only in special cases has steric retardation been found; steric acceleration does not play any detectable part in the acyclic series. It is concluded that the rate-determining step is the formation of a carbonium ion by way of a hydride ion transfer from the hydrocarbon molecule to an oxygen atom of the oxidizing agent.

Prof. K. B. Wiberg (University of Washington) from a study of the rate of oxidation of diphenylmethane and of its nuclear substituted derivatives in 95 per cent acetic acid concluded that the oxidation proceeds by initial removal of a hydrogen atom to give a benzhydryl radical which is then oxidized directly to benzophenone. In support of such a mechanism, oxidation of optically active 3-methylhexane gives an optically active tertiary alcohol, a result which seems to exclude the initial formation of a carbonium ion. The formation of camphenilanic acid by the oxidation of isocamphane is also cited in support of that view. Further evidence of an indirect nature is derived from a study of the action of chromyl chloride on propylbenzene- $\beta \beta \mathrm{d}_{2} ;$ benzyl methyl ketone is among the products, and it was found to have one deuterium atom in the $\alpha$-position, indicating a deuterium shift during the reaction.

The oxidation of tertiary paraffins by chromic acid in presence of sulphuric acid is known to lead to tertiary alcohols, and the course of the reaction can be interpreted in terms of dehydration of the alcohol to olefin. In a study of the oxidation by Dr. W. J. Hickinbottom (Queen Mary College, London) the conditions were selected so that tertiary alcohols could not be formed, by using chromium trioxide in acetic anhydride. Under these conditions, paraffins gave products which were qualitatively identical with those from the corresponding olefins in presence of weak acids. Further, some of the paraffins gave unsaturated products. From these results and from quantitative measurements of the rates of oxidation it was concluded that tertiary paraffins are attacked preferentially at the tertiary carbon atom with the subsequent formation of an olefin.

A possible key to the oxidation of paraffins by chromic acid may lie in the behaviour of the alcohols which may be derived from them by oxidation. Our knowledge of the course of the oxidation of alcohols is based on the work of Prof. F. H. Westheimer (Harvard University). In continuation of this work, he described, with Y. E. Chang, a study of the oxidation of pinacol and its monomethyl ether. A feature of the pinacol oxidation is that it proceeds 2.7 times as fast in deuteriun oxide as in water. This was interpreted to mean that the hydroxyl bond is not cleaved in the rate-controlling step of the oxidation. The relative merits of an ester mechanism and hydride abstraction were discussed and many useful ideas exchanged.

Dr. W. A. Waters (Oxford) reviewed the relationship between vanadate and permanganate oxidations. The role of trivalent manganese and of organic freeradical intermediates in permanganate oxidations was reviewed. Features diagnostic of one-electron oxidations were brought to notice. It was stressed that lack of diagnostic evidence need afford no grounds for the rejection of a reaction mechanism.

Dr. J. W. Ladbury (I.C.I., Ltd., Plastics Division, Welwyn Garden City) and Dr. C. F. Cullis (Imperial College of Science and Technology, London) discussed the oxidation of inorganic and organic compounds by permanganate. The development of reactionrate with time depends on the nature of the compound undergoing oxidation. The observed types of behaviour fall, broadly speaking, into four categories according to the shape of the reaction - time curves. Thus there may be: (1) a continuous decrease in rate with time, usually not strictly according to a 